\title{
Supplementary Information: Goslin - A Grammar of Succinct Lipid Nomenclature
}

Dominik Kopczynski ${ }^{1, *}$, Nils Hoffmann ${ }^{1, *}$, Bing Peng ${ }^{1,2}$ and

Robert Ahrends ${ }^{3, \otimes}$

${ }^{1}$ Leibniz-Institut für Analytische Wissenschaften - ISAS - e.V., 44139 Dortmund, Germany

2Division of Rheumatology, Department of Medicine, Solna,

Karolinska Institutet and Karolinska University Hospital, 17176

Stockholm, Sweden

${ }^{3}$ University of Vienna, Department of Analytical Chemistry, 1090

Vienna, Austria

*Shared first authors

${ }^{\otimes}$ Robert.Ahrends@univie.ac.at

\section{Contents}

1 Web Application and REST API 2

2 C++ Implementation 9

3 Python Implementation $\quad 12$

4 R Implementation $\quad 16$

5 Java Implementation $\quad 19$

6 Goslin Object Model 23

7 List of Supported Lipids $\quad 24$ 


\section{Web Application and REST API}

\section{Interactive Usage}

The interactive grammar of succinct lipid nomenclatures (Goslin) web application is available at https://apps.lifs.isas.de/goslin. It provides two forms to i) upload a file containing one lipid name per line (see Supplementary Figure S1), or ii) upload a list of lipid names, defined by the user in an interactive form (see Supplementary Figure S2). The latter form also allows pasting lists of lipid names directly from the clipboard with CTRL+V. Both forms provide feedback for issues concerning every processed lipid, such as invalid names or typos (see Supplementary Figure S3), to allow the user to cross-check their data before proceeding.

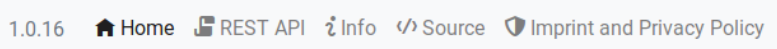

Goslin webapplication - A Parser, Validator and Normalizer for Lipid Nomenclature

jGoslin is a parser implementation for lipid species and subspecies names following the shorthand notation for lipids and fragments with some improvements concerning plasmanyls \& plasmenyls to avoid ambiguities with fatty acid chain double bond counting and the LipidMaps Nomenclature.

We collaborate with the Lipidomics Standards Initiative concerning lipid nomenclature used for reporting of lipid species identifications.

The parser implementation is based on the Goslin grammars and returns a classification of the parsed lipid name including its perceived level (e.g. structural subspecie), including fatty acid and long chain base details, if they are deducable from the provided string. The hierarchy levels and the names are consistent with those used in the shorthand notation for lipids publication as well as in SwissLipids.

LIFS is a project funded by BMBF as part of the German network for bioinformatics infrastructure (de.NBI) to provide and support tools for lipid-related bioinformatics.

Parse, Translate \& Validate Lipid Names

Upload a text file with one lipid name per row

File with lipid names to validate

Browse... No file selected.

Select a local file to upload and validate.

Figure S1: Goslin web application submission form for text files with one lipid name per row. 
Or enter individual lipid names

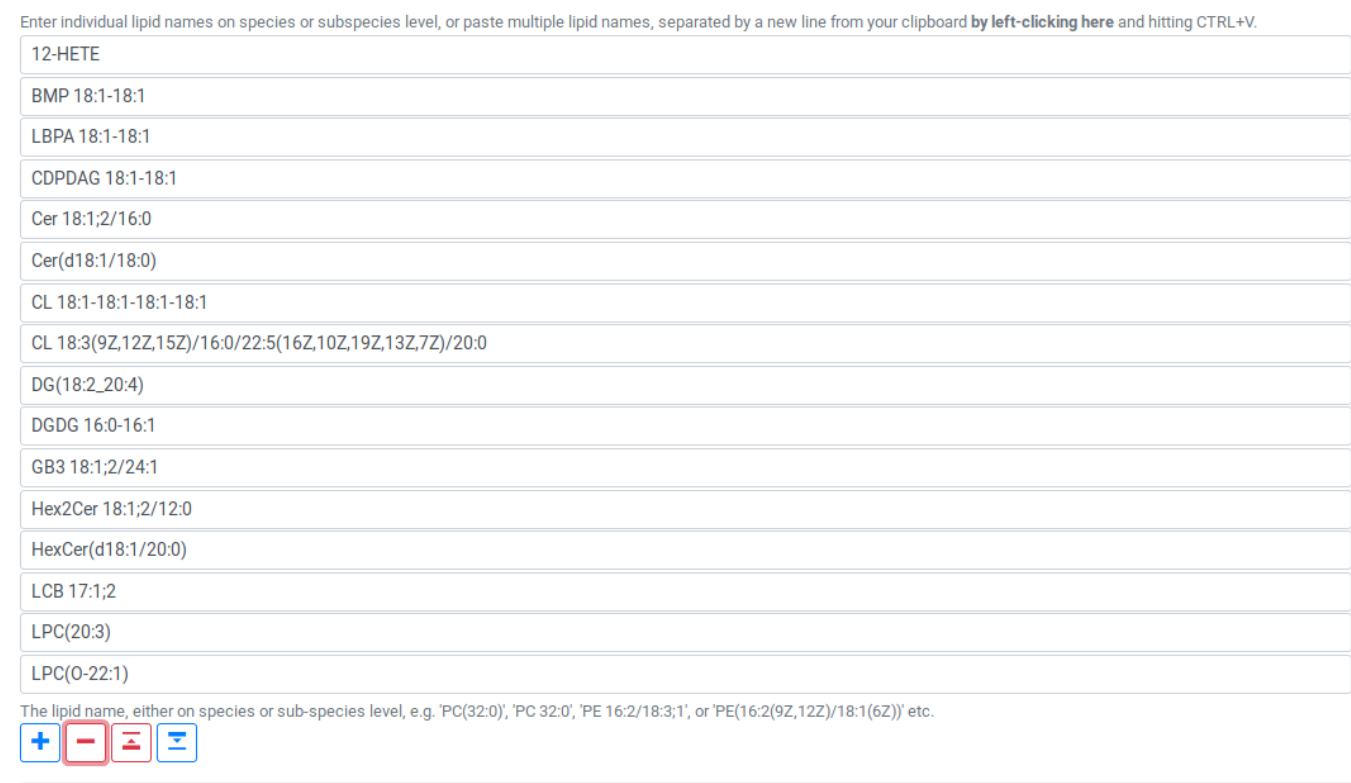

Figure S2: Goslin web application submission form for user-defined lipid names.

\section{Or enter individual lipid names}

Enter individual lipid names on species or subspecies level, or paste multiple lipid names, separated by a new line from your clipboard by left-clicking here and hitting CTRL+V

$$
\text { SG 16:2/18:1 }
$$

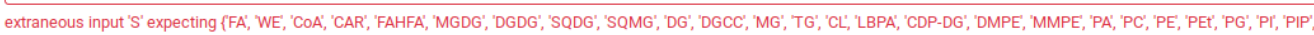

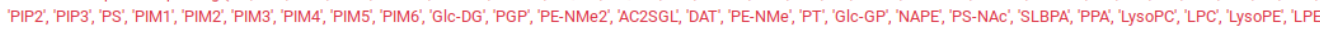

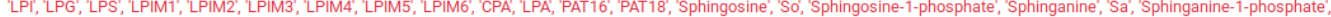

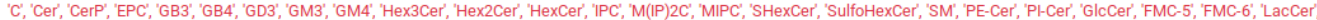

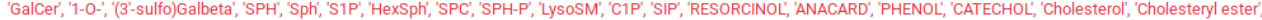

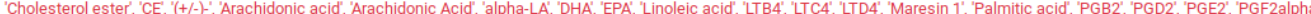

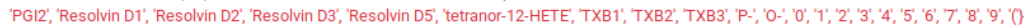

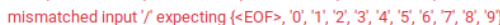

The lipid name, either on species or sub-species level, e.g. 'PC(32:0), 'PC 32:0', 'PE 16:2/18:3;1', or 'PE(16:2(9Z, 12Z)/18:1(6Z))' etc.

$+-\bar{z}$

Figure S3: Goslin web application submission form for user-defined lipid names provides feedback for unknown or unsupported names and parts thereof. 


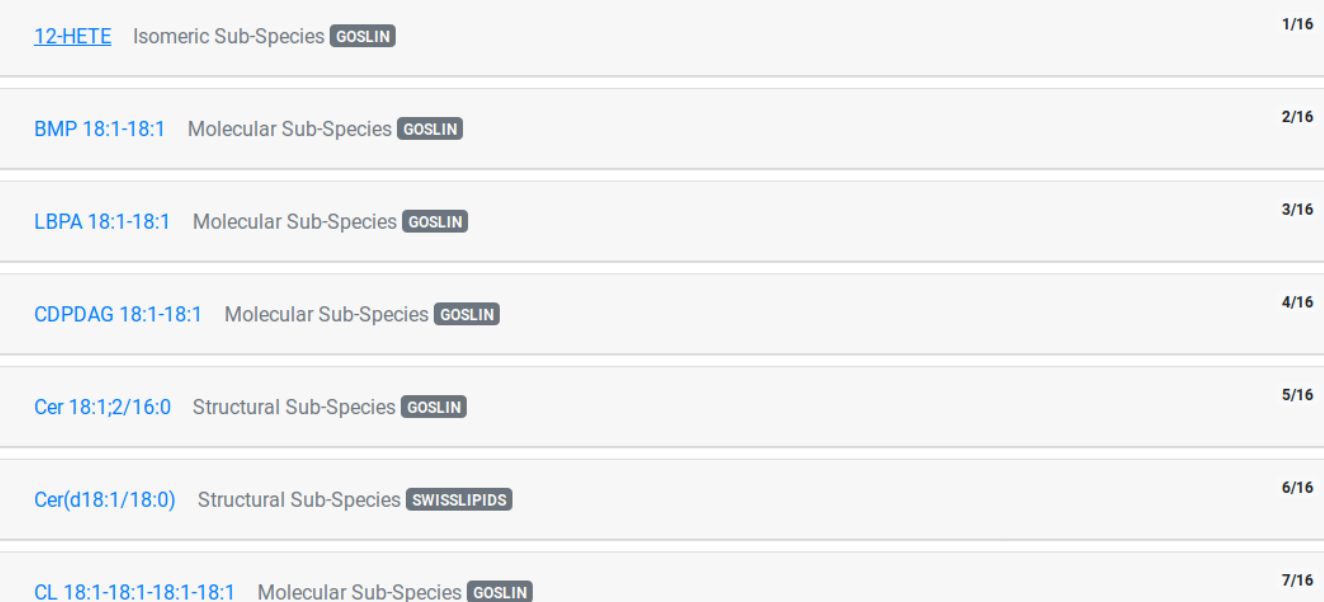

\begin{tabular}{ll} 
CL 18:1-18:1-18:1-18:1 Molecular Sub-Species GoSLIN $\quad 7 / 16$ \\
\hline
\end{tabular}

CL 18:3(9Z,12Z,15Z)/16:0/22:5(16Z,10Z,19Z,13Z,7Z)/20:0 Isomeric Sub-Species GosLIN

DG(18:2_20:4) Molecular Sub-Species SWISSLIPIDS $\quad 9 / 16$

DGDG 16:0-16:1 Molecular Sub-Species GosLIN $\quad 10 / 16$

GB3 18:1;2/24:1 Structural Sub-Species GosLIN $\quad 11 / 16$

Hex2Cer 18:1;2/12:0 Structural Sub-Species GosLIN $\quad 12 / 16$

Figure S4: Parsing results are displayed as 'cards' for every lipid name. Clicking on a card opens it and shows details of the according lipid.

After successful validation, the validated lipids are returned in overview cards (see Supplementary Figure S4), detailing their LipidMAPS classification ${ }^{1}$, cross-links to SwissLipids ${ }^{2}$ and/or LipidMAPS or $\mathrm{HMDB}^{3}$. Additionally, the cards show summary information about the number of carbon atoms, double bonds, hydroxylations and detailed information, such as double bond position, long-chain-base status, and the bond type of the fatty acyl to the head group for each fatty acyl, if available (see Supplementary Figure S5) . 


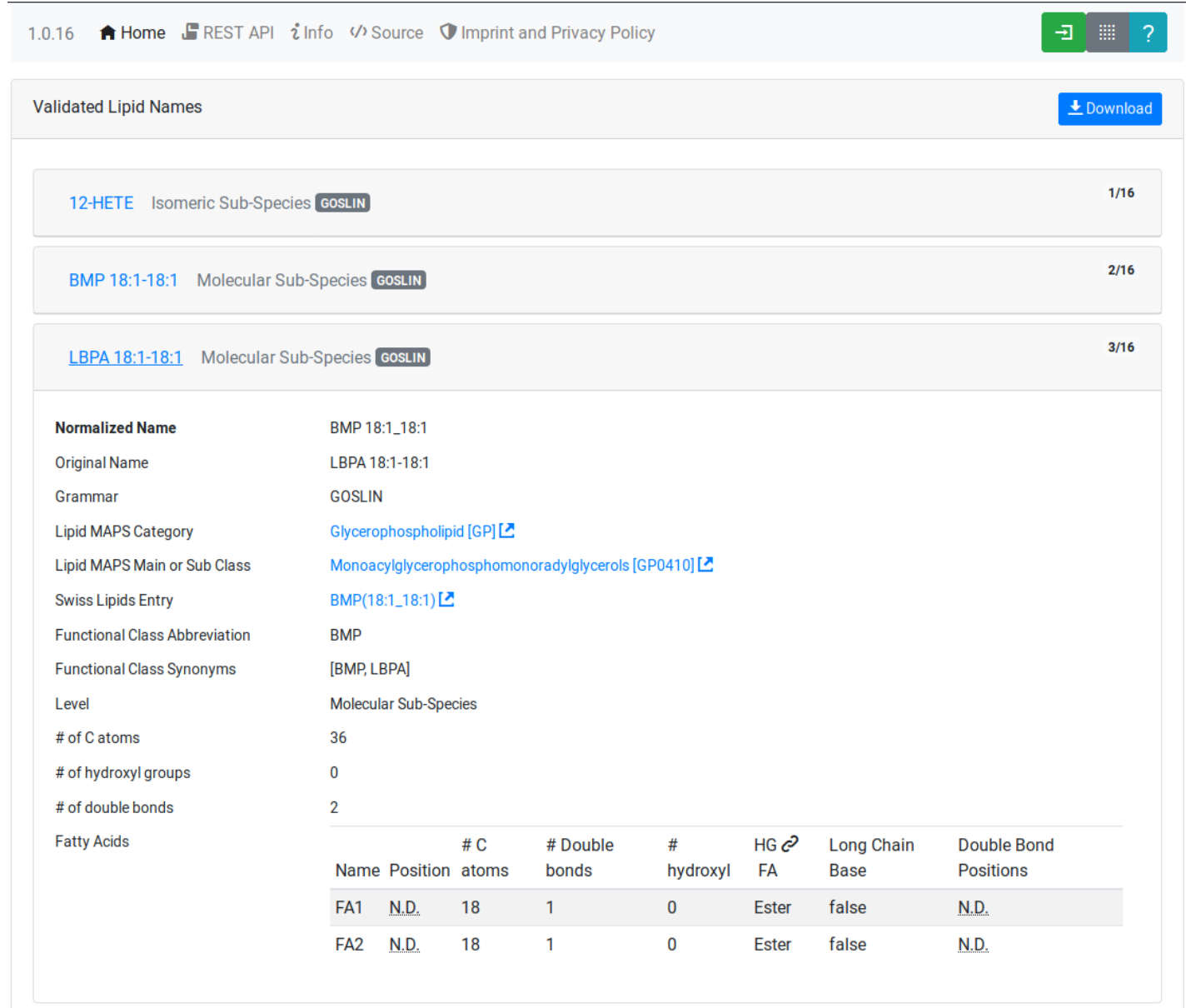

Figure S5: Each result card displays summary and detail information about a lipid. Depending on the lipid level, this can include information about each individual fatty acyl. Cross-links to SwissLipids and LipidMAPS are shown where a normalized lipid name could be matched unambiguously against the normalized shorthand names of SwissLipids and / or LipidMAPS lipids.

The source code for the web application and instructions to build it as a Docker container are available at https://github.com/lifs-tools/goslin-webapp under the terms of the open source Apache license version 2. 


\section{Programmatic access via the REST API}

An interactive documentation for the representational state transfer (REST) application programming interface (API) of the Goslin web application is available at https: //apps. lifs. isas. de/goslin/swagger-ui.html (see Supplementary Figure S6). To illustrate its usage, we will briefly show a small example how a user can access the REST API with a standard hypertext transfer protocol (HTTP) client.

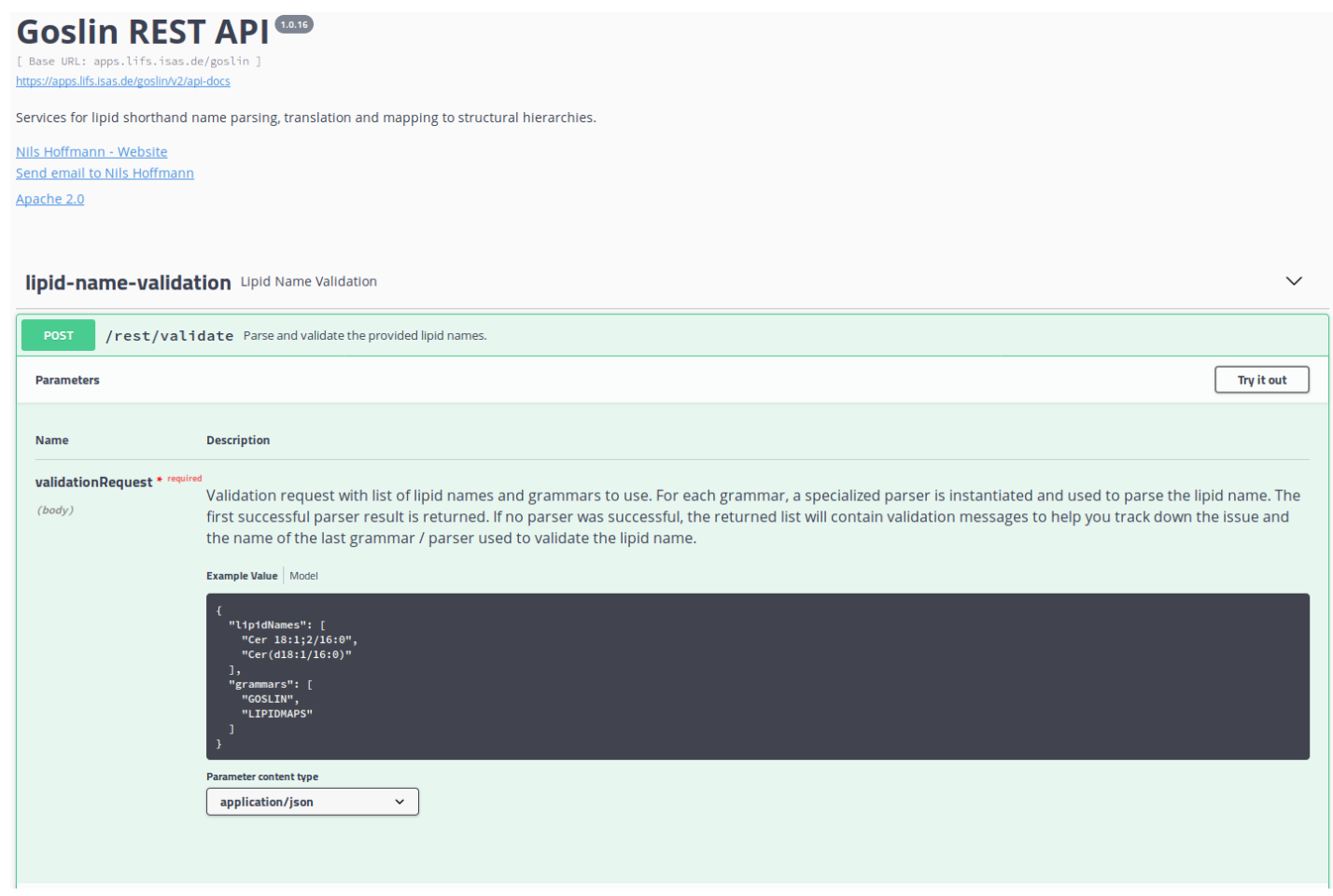

Figure S6: The Goslin web application provides an interactive documentation for its REST API to simplify programmatic access.

The Structure for the request consists of a JavaScript object notation (JSON) object \{\} enclosing two lists, with the names lipidNames and grammars. Acceptable values for grammars are: LIPIDMAPS, GOSLIN, GOSLIN_FRAGMENTS, SWISSLIPIDS, and HMDB. A complete list is available from the interactive REST API documentation's Models section under ValidationRequest. Both fields in the ValidationRequest accept commaseparated entries, enclosed in double quotes:

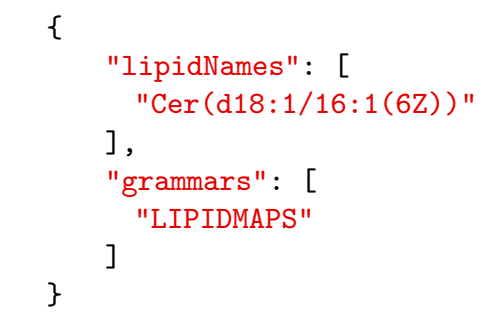

Sending the HTTP POST request with curl as an HTTP client looks as follows:

curl -X POST "https://apps.lifs.isas.de/goslin/rest/validate" -H "accept: */*" -H 
The REST API will return the following result for the request, with a HTTP response code of 200 (OK). This result returns a map of properties for each lipid name that was parsed. If at least one name is not parseable, the REST API will return a response code of 400 (Client error), together with the same results reponse object. In that case, the failedToParse field in the response will contain the number of lipid names that could not be parsed. For those results where no grammar was applicable, the grammar field will contain the string NOT_PARSEABLE. In other cases, that field will contain the last grammar used to parse the lipid name and the messages field will contain a list of validation messages that help to narrow down the offending bits in the lipid name.

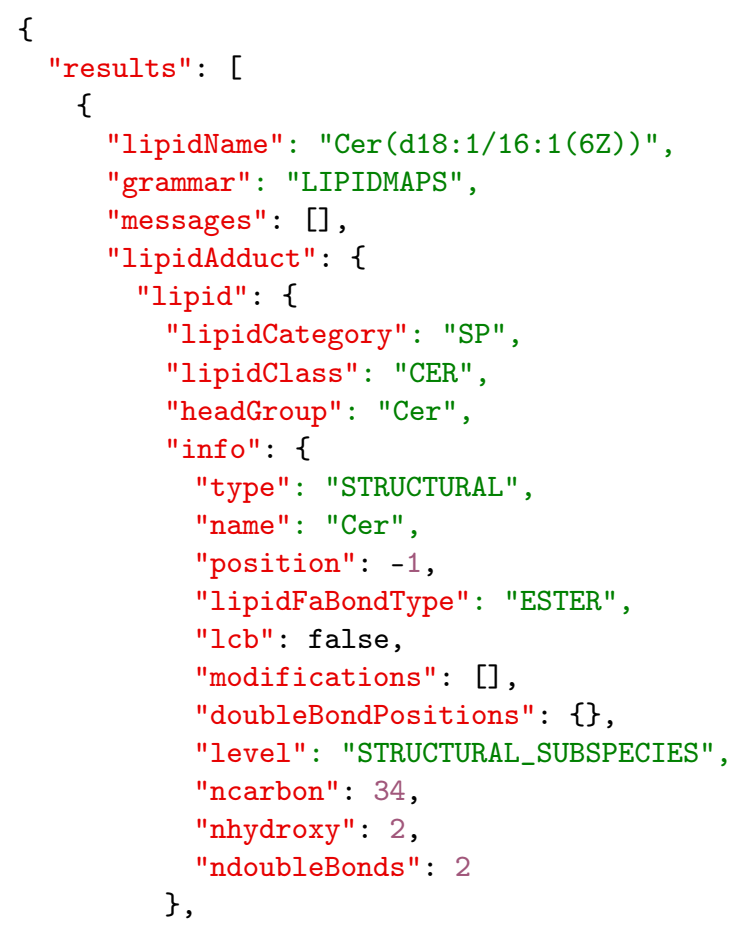

The response part also reports the normalized name (goslinName), as well as classification information using the LipidMAPS category and class associated to the parsed lipid.

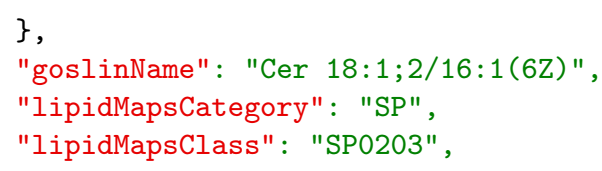

The response also reports information on the fatty acyls detected in the lipid name. In this case, a long chain base (LCB) (in the ceramide) has been detected. The name given here as an example was classified on structural subspecies level, since the LCB contains one double bond, but without positional $E / Z$ information. The fatty acyl FA1 at the sn2 position does report $E / Z$ information for its double bond, thus FA1 is an isomeric fatty acyl. Overall, the lipid can thus be classified as a structural subspecies.

"fattyAcids": \{ 


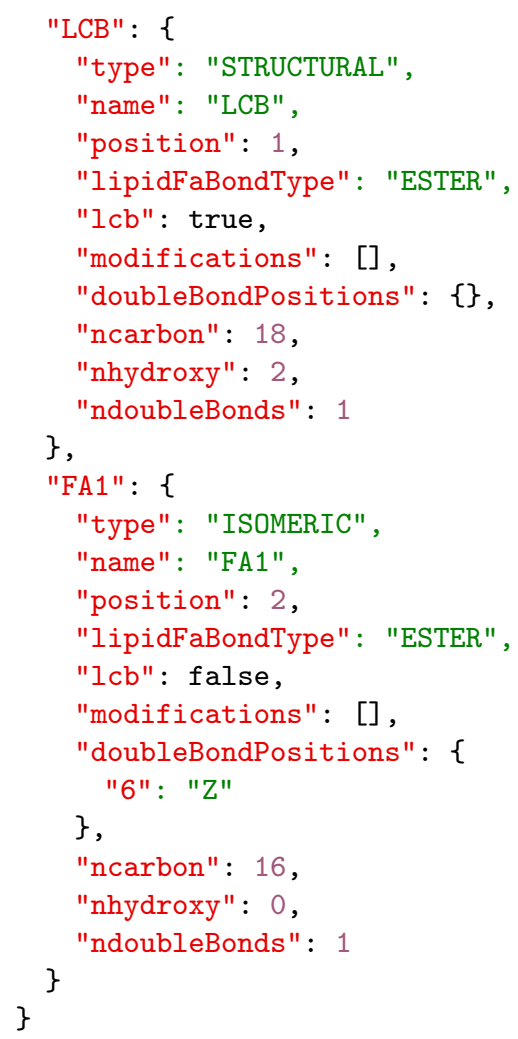

Finally, the response reports the total number lipid names received, the total number parsed and the total number of parsing failures.

],

"totalReceived": 1,

"totalParsed": 1 ,

"failedToParse": 0 \} 


\section{C++ Implementation}

This is the documentation for the Goslin reference implementation for $\mathrm{C}_{++}$. Please be aware, that the documentation is dedicated to developers of tools for computational lipidomics who want to use cppgoslin within their project. If you are interested to run Goslin as a user, please read Supplementary Section 1. The cppgoslin implementation has been developed with the following objectives:

1. To ease the handling with lipid names for developers working on mass spectrometry-based lipidomics tools.

2. To offer a tool that unifies all existing dialects of lipid names.

It is an open-source package under the MIT License available via github ${ }^{1}$. For a detailed structure of the implementation, read Supplementary Section 6.

\section{Prerequisites}

The cppgoslin library needs a GNU g++ compiler version with support for the $\mathrm{C}++11$ standard. It comes with simple makefiles for easy compilation and installation. You need the following packages:

$\$ \mathrm{~g}^{++}$(compiler)

To install the library globally on your system, simply type:

\$ [sudo] make install

Be sure that you have root permissions. Here, the library and headers are installed into the /usr directory. If you want to change that location, you have to edit the first line within the makefile.

\section{Testing cppgoslin}

We set up more than 150000 single unit and integration tests, to ensure that cppgoslin is parsing correctly. To run the tests, please type:

$\$$ make test

$\$$ make runtests

If a test should fail, please contact the developers ${ }^{2}$.

\footnotetext{
${ }^{1}$ https://github.com/lifs-tools/cppgoslin

${ }^{2}$ goslin@lipidomics . at
} 


\section{Using cppgoslin}

The two major functions within cppgoslin are the parsing and printing of lipid names. A minimalistic example will demonstrate both functions the easiest way. In the examples folder, you will find the lipid_name_parser.cpp file. Compile it by typing:

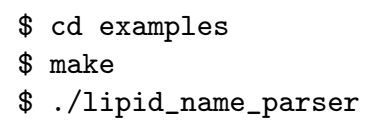

Here is the minimalistic $\mathrm{C}++$ code:

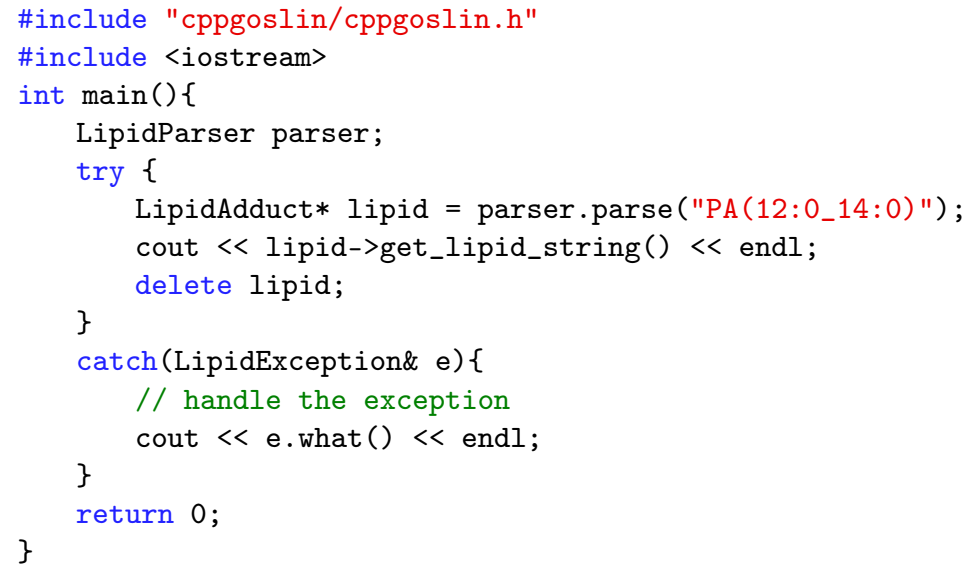

To handle unexpected behavior, the parsing command should always be placed within a try/catch block and the LipidAdduct pointer should be deleted after usage to avoid memory leaks. Be aware when changing the installation directory, you also have to change the library directory within the examples makefile.

To retrieve a parsed lipid name on a higher hierarchy of lipid level, simply define the level when requesting the lipid name:

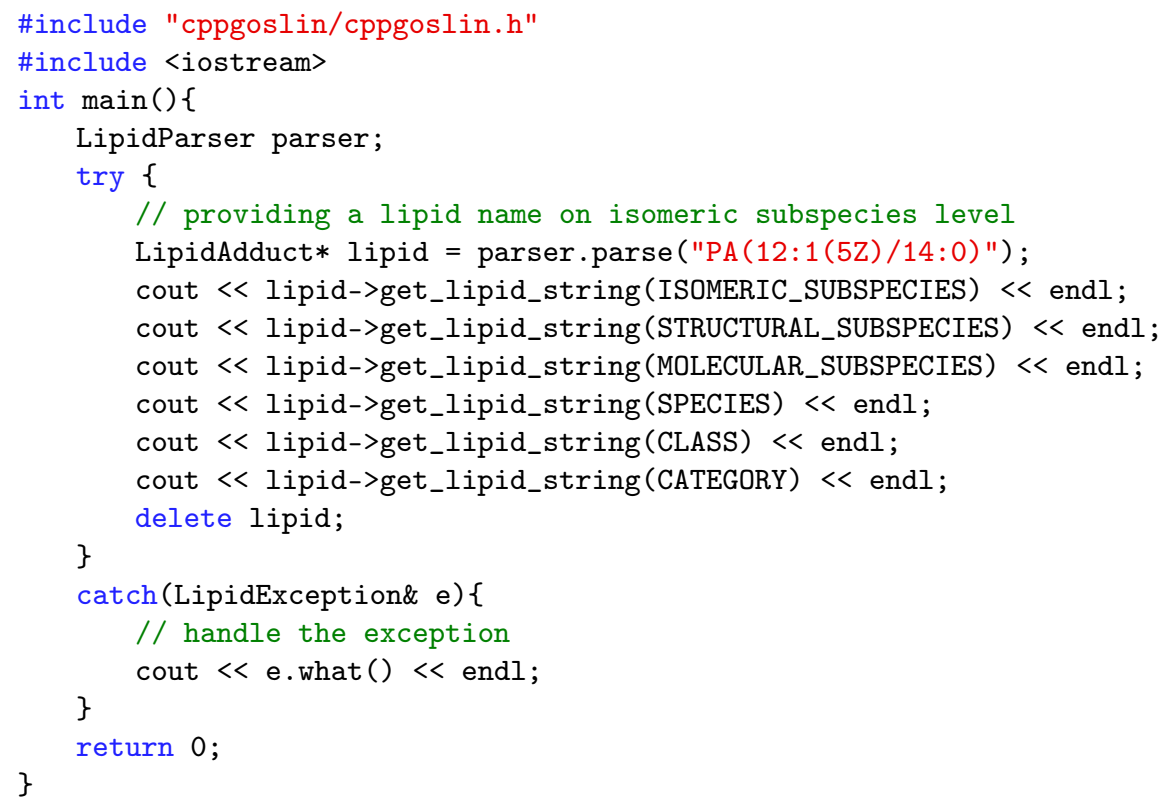


Requesting a lipid name on a lower level than the provided will throw an exception. This functionality especially enables an easy way for computing data for histograms on lipid class or category level.

To increase the parsing performance, one can pick a parser for only one specific grammar:

GoslinParser goslin_parser;

GoslinFragmentParser goslin_fragment_parser;

LipidMapsParser lipid_maps_parser;

SwissLipidsParser swiss_lipids_parser;

HmdbParser hmdb_parser; 


\section{Python Implementation}

This is the documentation for the Goslin reference implementation for Python 3. Please be aware, that the documentation is dedicated to developers of tools for computational lipidomics who want to insert pygoslin into their project. If you are interested to run Goslin as a user, please read Section 1. The pygoslin implementation has been developed with the following objectives:

1. To ease the handling with lipid names for developers working on mass spectrometrybased lipidomics tools.

2. To offer a tool that unifies all existing dialects of lipid names.

It is an open-source package under the MIT License available via github ${ }^{3}$. For a detailed structure of the implementation, read Supplementary Section 6.

\section{Prerequisites}

The pygoslin package uses Python's package management system pip to create an isolated and defined build environment. You need Python $>=3.5$ and the following packages to build the pygoslin package:

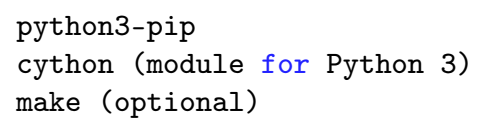

To install the package globally in your Python distribution, simply type:

\$ [sudo] make install

or

\$ [sudo] python setup.py install

Be sure that you have root permissions.

\section{Testing pygoslin}

We set up more than 150000 single unit and integration tests, to ensure that pygoslin is parsing correctly. To run the tests, please type:

$\$$ make test

or

${ }^{3}$ https://github.com/lifs-tools/pygoslin 
try:

lipid $=$ parser $\cdot$ parse (lipid_name $)$

print (lipid.get_lipid_string (LipidLevel.ISOMERIC_SUBSPECIES))

print (lipid.get_lipid_string (LipidLevel.STRUCTURAL_SUBSPECIES))

print (lipid.get_lipid_string (LipidLevel.MOLECULAR_SUBSPECIES))

print (lipid.get_lipid_string (LipidLevel.SPECIES))

print (lipid.get_lipid_string (LipidLevel.CLASS))

print(lipid.get_lipid_string (LipidLevel.CATEGORY))

except Exception as e:

print (e)

This functionality especially enables an easy way for computing data for histograms on lipid class or category level. Requesting a lipid name on a lower level than the provided will raise an exception. 


\section{R Implementation}

This project is a parser, validator and normalizer implementation for shorthand lipid nomenclatures, using the Grammar of Succinct Lipid Nomenclatures project for the $\mathrm{R}$ language ${ }^{4}$.

Goslin defines multiple grammars compatible with ANTLRv4 for different sources of shorthand lipid nomenclature. This allows to generate parsers based on the defined grammars, which provide immediate feedback whether a processed lipid shorthand notation string is compliant with a particular grammar, or not.

rgoslin uses the Goslin grammars and the cppgoslin parser to support the following general tasks:

1. Facilitate the parsing of shorthand lipid names dialects.

2. Provide a structural representation of the shorthand lipid after parsing.

3. Use the structural representation to generate normalized names.

rgoslin is an open-source package available via github ${ }^{5}$.

\section{Prerequisites}

This project uses the $\mathrm{R}$ programming language. To be able to use it, please install $\mathrm{R}^{6}$ following the instructions for your particular operating system. rgoslin is based on native $\mathrm{C}_{++}$code (via cppgoslin). It therefore requires additional tools on your system to compile and install it. Please see the Rcpp FAQ ${ }^{7}$, question 1.3 for installation details for your specific operating system.

Install the 'devtools' package with the following command.

if (!require(devtools)) \{ install.packages ("devtools") \}

Run

install_github("lifs-tools/rgoslin")

to install from the github repository.

This will install the latest, potentially unstable development version of the package with all required dependencies into your local $R$ installation.

If you want to use a proper release version, referenced by a Git tag (here: v1.0.0) install the package as follows:

\footnotetext{
${ }^{4}$ https://www.r-project.org/

${ }^{5}$ https://github.com/lifs-tools/rgoslin

${ }^{6}$ https://cloud.r-project.org/

${ }^{7}$ https://cran.r-project.org/web/packages/Rcpp/vignettes/Rcpp-FAQ.pdf
} 
If you have cloned the code locally, use devtools as follows. Make sure you set the working directory to where the API code is located. Then execute

library (devtools)

install (".")

\section{Testing rgoslin}

rgoslin uses the testthat $R$ package to provide unit tests for the lipid name parsing methods. The tests are located in the tests folder. To run the tests, execute

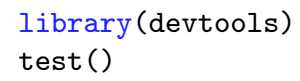

\section{Using rgoslin}

To load the package, start an $\mathrm{R}$ session and type

library (rgoslin)

Type the following to see the package vignette / tutorial:

vignette('introduction', package = 'rgoslin')

In order to use the provided translation functions of rgoslin, you first need to load the library.

library (rgoslin)

To check, whether a given lipid name can be parsed by any of the parsers supplied by cppgoslin, you can use the isValidLipidName method. It will return TRUE if the given name can be parsed by any of the available parsers and FALSE if the name was not parseable.

| isValidLipidName("PC $32: 1 "$ )

Using parseLipidName with a lipid name returns a named vector of properties of the parsed lipid name.

pc32vector <- parseLipidName("PC 32:1")

pc32df <- as.data.frame(t(pc32vector))

If you want to set the grammar to parse against manually, this is also possible:

originalName <- "TG(16:1(5E)/18:0/20:2(3Z,6Z))"

tagVec <- rgoslin: :parseLipidNameWithGrammar (originalName, "LipidMaps")

tagDf <- as.data.frame $(t(\operatorname{tagVec}))$ 
Currently, the following grammars are available: LipidMaps, SwissLipids, Goslin, GoslinFragments, HMDB.

If you want to parse multiple lipid names, use the parseLipidNames method with a vector of lipid names. This returns a data frame of properties of the parsed lipid names with one row per lipid.

| multipleLipidNames <- parseLipidNames(c("PC 32:1","LPC 34:1", "TG(18:1_18:0_16:1)"))

Finally, if you want to parse multiple lipid names and want to use one particular grammar:

originalNames <- c("PC 32:1", "LPC 34:1","TAG 18:1_18:0_16:1")

multipleLipidNamesWithGrammar <- parseLipidNamesWithGrammar (originalNames, "Goslin") 


\section{Java Implementation}

This project is a parser, validator and normalizer implementation for shorthand lipid nomenclatures, based on Goslin for the Java programming language ${ }^{8}$.

Goslin defines multiple grammars compatible with ANTLRv4 for different sources of shorthand lipid nomenclature. This allows to generate parsers based on the defined grammars, which provide immediate feedback whether a processed lipid shorthand notation string is compliant with a particular grammar, or not.

Here, jgoslin uses the Goslin grammars and the generated parsers to support the following general tasks:

1. Facilitate the parsing of shorthand lipid names dialects.

2. Provide a structural representation of the shorthand lipid after parsing.

3. Use the structural representation to generate normalized names.

Furthermore, jgoslin is an open-source package available via github ${ }^{9}$.

\section{Prerequisites}

This project is based on Java 11. To use it, you need a Java Runtime Environment (JRE) installed on your system. If you want to use the library in your own Java projects, you need a Java Development Kit (JDK) installed on your system. Please consult https://adoptopenjdk.net/installation.html for installation options and instructions for your operating system.

Installation instructions

Building the project and generating client code from the command-line

In order to build the client code and run the unit tests, execute the following command from a terminal:

./mvnw install

or on Windows:

mvnw.bat install

This compiles and tests the Java library.

\footnotetext{
${ }^{8}$ https://go.java/

${ }^{9}$ https://github.com/lifs-tools/jgoslin
} 


\section{Testing jgoslin}

Here, jgoslin comes with a comprehensive collection of unit (JUnit 5), integration (JUnit 5) and acceptance (Cucumber) tests. You can run all of them as follows:

./mvnw verify

\section{Using the command-line interface}

The cli sub-project provides a command line interface (CLI) for parsing of lipid names either from the command line or from a file with one lipid name per line.

After building the project as mentioned above with ./mvnw install, the cli/target folder will contain the jgoslin-cli-<VERSION $>$-bin.zip file. Alternatively, you can download the latest cli zip file from Bintray: https://bintray.com/lifs/maven/jgoslin-cli[Search for latest jgoslin-cli-<VERSION>-bin.zip artefact] and click to download.

In order to run the validator, unzip that file, change into the unzipped folder and run

java -jar jgoslin-cli-<VERSION>.jar

to see the available options.

To parse a single lipid name from the command line using all available parsers, run java -jar jgoslin-cli-<VERSION>.jar -n "Cer (d18:1/20:2)"

The output will tell you what is done and will echo a table of the results to the terminal:

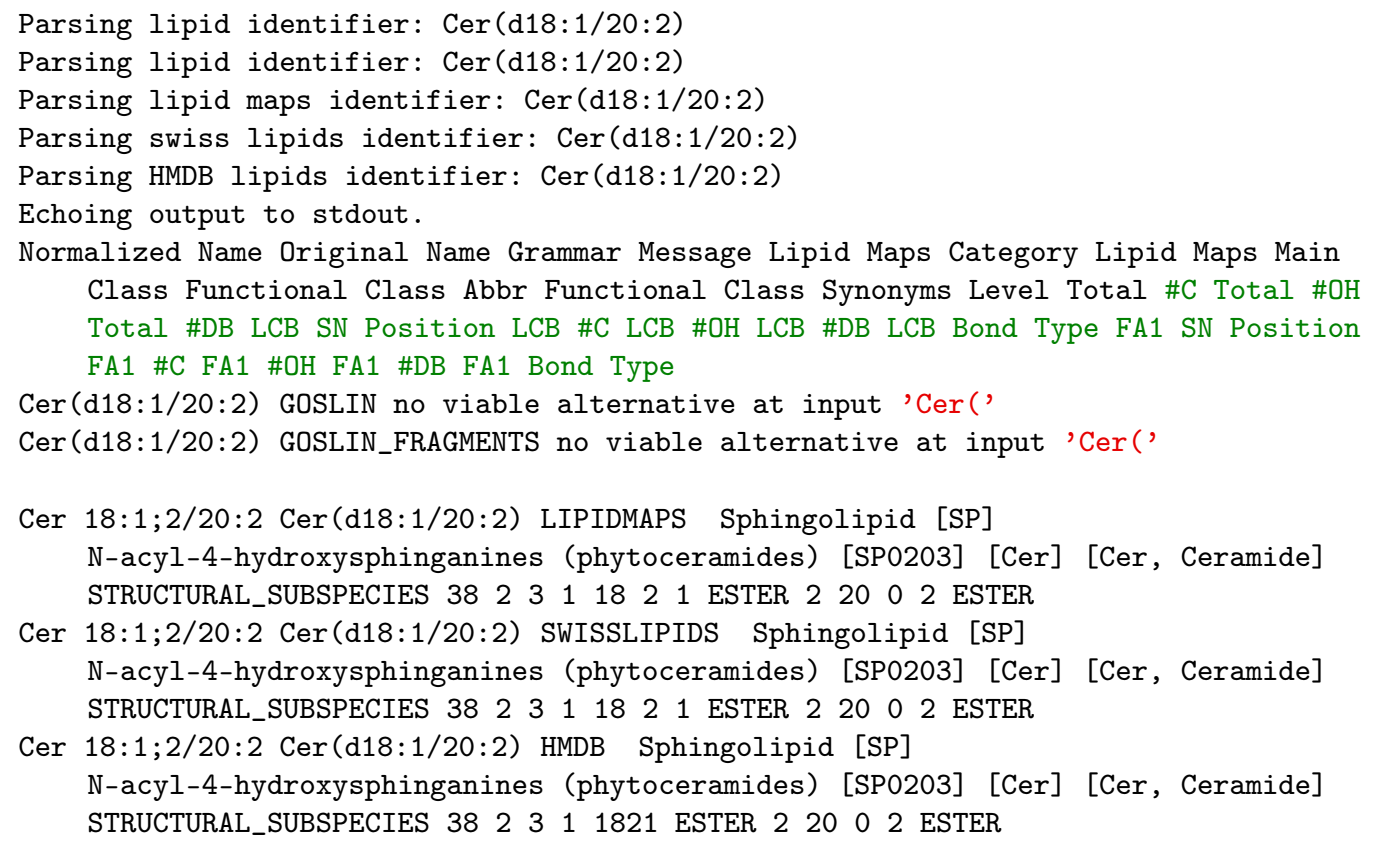

To parse multiple lipid names from a file via the commmand line, run 
java -jar jgoslin-cli-<VERSION $\rangle$.jar -f lipidNames.txt

To use a specific grammar, instead of trying all, run

java -jar jgoslin-cli-<VERSION $>$.jar -f lipidNames.txt -g GOSLIN

To write output to the tab-separated output file 'goslin-out.tsv' instead of to the terminal, run

| java -jar jgoslin-cli-<VERSION>.jar -f lipidNames.txt -g GOSLIN -o

If you want to use all available grammars, simply omit the -g GOSLIN argument. Please note that you will then receive $N$ times $M$ lines in the output file, where $N$ is the number of lipid names and $\mathrm{M}$ the number of grammars.

\section{Using jgoslin}

To integrate jgoslin in your own projects as a library, please see the README file at https://github.com/lifs-tools/jgoslin for more details.

The following snippet shows how to parse a shorthand lipid name with the different parsers:

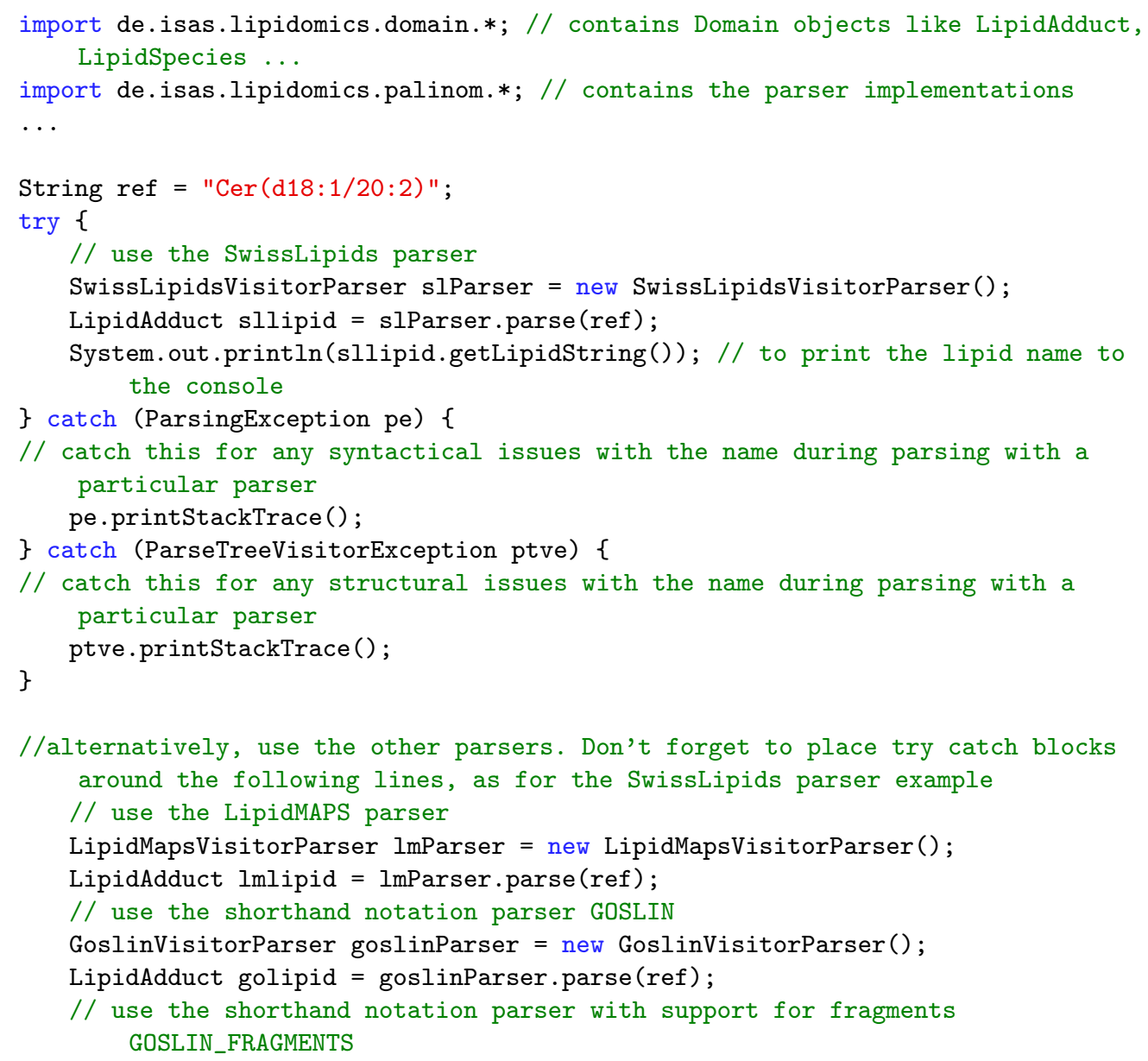




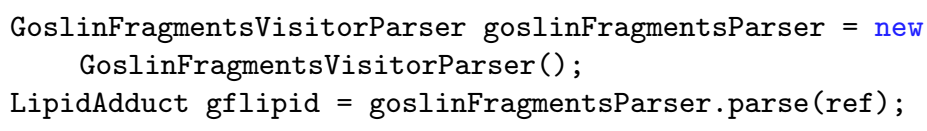

To retrieve a parsed lipid name on a higher hierarchy of lipid level, simply define the level when requesting the lipid name:

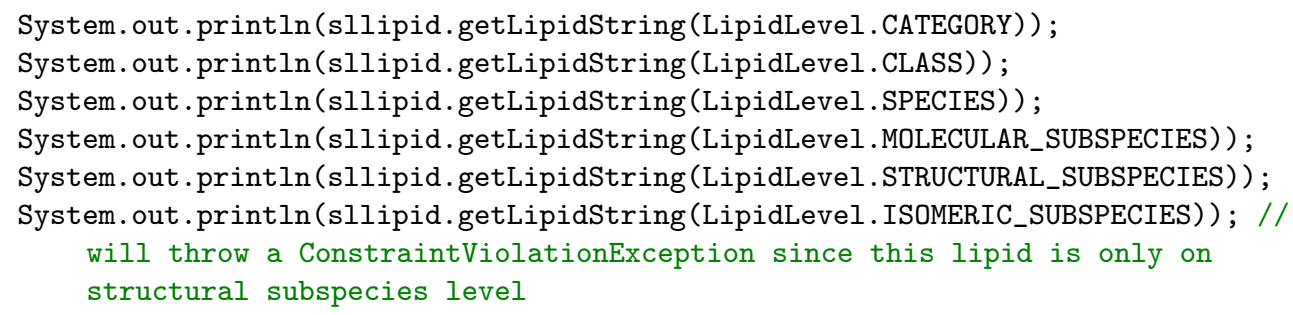

This functionality allows easy computation of aggregate statistics of lipids on lipid class, category or arbitrary levels. Requesting a lipid name on a lower level than the provided will raise an exception.

For an overview of the domain model used by jgoslin, please see Supplementary Section 6. 


\section{Goslin Object Model}

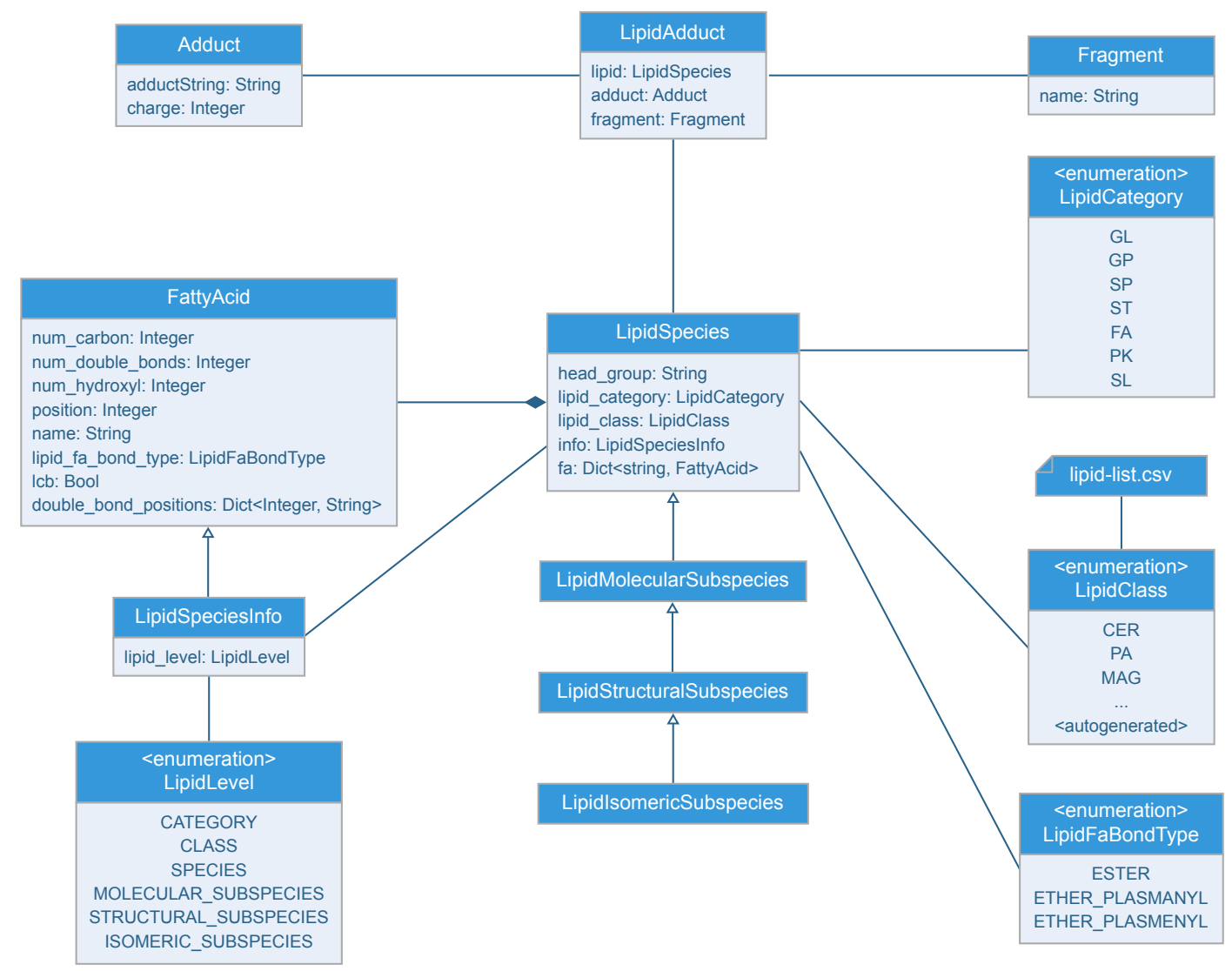

Figure S7: Goslin object model.

All goslin implementations are implementing the goslin object model as illustrated in Supplementary Figure S7. The classes LipidCategory, LipidLevel, LipidClass, and LipidFaBondType are predefined enumerations. Here, LipidClass is being generated automatically from a list containing lipid information (name, description, category, abbreviation, synonyms) for all implementations, see Supplementary Table S1 for details. This especially eases the maintenance and ensures that the goslin implementations have the same data base. The main class unifying all classes and being provided by the parsers is LipidAdduct. It contains information about the pure lipid, the adduct as well as the fragment (if defined). The different lipid classes inherit from each other in a hierarchical fashion as defined by Liebisch et al. ${ }^{4}$. A dictionary with the class LipidSpecies is storing all its associated fatty acyl chains which are defined within the class FattyAcid. For storing the cummulated information on species level for the carbon length, double bonds, etc, the class LipidSpeciesInfo is utilized. 


\section{List of Supported Lipids}

Table S1: List of supported lipids, lipid classes and their normalized abbreviations

\begin{tabular}{|c|c|c|}
\hline Category & Description & Abbreviation \\
\hline \multirow{42}{*}{ Fatty acyls } & Other Docosanoids & 10-HDoHE \\
\hline & Epoxyeicosatrienoic acids & 11(12)-EET \\
\hline & Hydroxy/hydroperoxyeicosatetraenoic acids & 11,12-DHET \\
\hline & Other Docosanoids & 11-HDoHE \\
\hline & Hydroxy/hydroperoxyeicosatetraenoic acids & 11-HETE \\
\hline & Other Octadecanoids & 12(13)-EpOME \\
\hline & Hydroxy/hydroperoxyeicosapentaenoic acids & 12-HEPE \\
\hline & Hydroxy/hydroperoxyeicosatetraenoic acids & 12-HETE \\
\hline & Hydroxy/hydroperoxyeicosatrienoic acids & 12-HHTrE \\
\hline & Fatty acids and conjugates & 12-OxoETE \\
\hline & Other Octadecanoids & 13-HODE \\
\hline & Other Octadecanoids & 13-HOTrE \\
\hline & Epoxyeicosatrienoic acids & 14(15)-EET \\
\hline & Other Eicosanoids & 14(15)-ЕрЕTE \\
\hline & Hydroxy/hydroperoxyeicosatetraenoic acids & 14,15-DHET \\
\hline & Hydroxy/hydroperoxyeicosapentaenoic acids & 15-HEPE \\
\hline & Hydroxy/hydroperoxyeicosatetraenoic acids & 15-HETE \\
\hline & Prostaglandins & 15d-PGJ2 \\
\hline & Other Docosanoids & 16-HDoHE \\
\hline & Hydroxy/hydroperoxyeicosatetraenoic acids & 16-HETE \\
\hline & Hydroxy/hydroperoxyeicosapentaenoic acids & 18-HEPE \\
\hline & Epoxyeicosatrienoic acids & 5(6)-EET \\
\hline & Hydroxy/hydroperoxyeicosatetraenoic acids & 5,12-DiHETE \\
\hline & Lipoxins & $5,6,15-L X A 4$ \\
\hline & Hydroxy/hydroperoxyeicosatetraenoic acids & 5,6-DiHETE \\
\hline & Hydroxy/hydroperoxyeicosapentaenoic acids & 5-HEPE \\
\hline & Hydroxy/hydroperoxyeicosatetraenoic acids & 5-HETE \\
\hline & Hydroxy/hydroperoxyeicosatetraenoic acids & 5-HpETE \\
\hline & Fatty acids and conjugates & 5-OxoETE \\
\hline & Epoxyeicosatrienoic acids & 8(9)-EET \\
\hline & Hydroxy/hydroperoxyeicosatetraenoic acids & 8,9-DHET \\
\hline & Other Docosanoids & 8-HDoHE \\
\hline & Hydroxy/hydroperoxyeicosatetraenoic acids & 8-HETE \\
\hline & Other Octadecanoids & 9(10)-EpOME \\
\hline & Hydroxy/hydroperoxyeicosapentaenoic acids & 9-HEPE \\
\hline & Hydroxy/hydroperoxyeicosatetraenoic acids & 9-HETE \\
\hline & Other Octadecanoids & 9-HODE \\
\hline & Other Octadecanoids & 9-HOTrE \\
\hline & Unsaturated fatty acids & $\mathrm{AA}$ \\
\hline & Fatty acyl carnitines & CAR \\
\hline & Fatty acyl CoAs & CoA \\
\hline & Unsaturated fatty acids & DHA \\
\hline
\end{tabular}




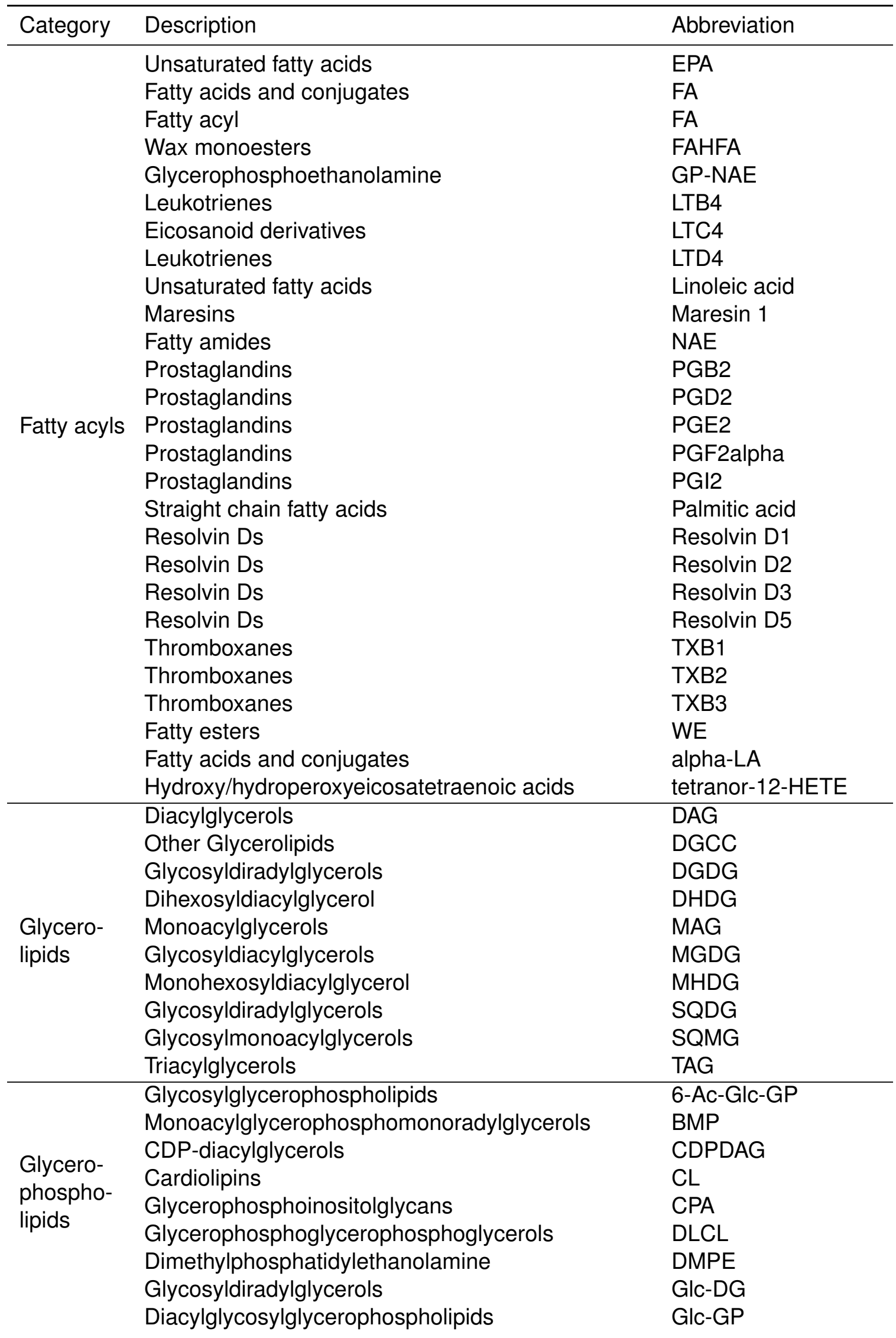




\begin{tabular}{|c|c|c|}
\hline Category & Description & Abbreviation \\
\hline \multirow{45}{*}{$\begin{array}{l}\text { Glycero- } \\
\text { phospho- } \\
\text { lipids }\end{array}$} & Lyso-CDP-diacylglycerol & LCDPDAG \\
\hline & Lysodimethylphosphatidylethanolamine & LDMPE \\
\hline & Lysomonomethylphosphatidylethanolamine & LMMPE \\
\hline & Monoacylglycerophosphates & LPA \\
\hline & Monoacylglycerophosphocholines & LPC \\
\hline & Monoacylglycerophosphoethanolamines & LPE \\
\hline & 1Z-alkenylglycerophosphoglycerols & $\mathrm{LPG}$ \\
\hline & Monoacylglycerophosphoinositols & LPI \\
\hline & Monoacylglycerophosphoinositolglycans & LPIM1 \\
\hline & Glycerophosphoinositolglycans & LPIM2 \\
\hline & Glycerophosphoinositolglycans & LPIM3 \\
\hline & Glycerophosphoinositolglycans & LPIM4 \\
\hline & Glycerophosphoinositolglycans & LPIM5 \\
\hline & Glycerophosphoinositolglycans & LPIM6 \\
\hline & $\begin{array}{l}\text { Lysophosphatidylinositol- mannosideinositolphos- } \\
\text { phate }\end{array}$ & LPIMIP \\
\hline & Lysophosphatidylinositol-glucosamine & LPIN \\
\hline & Monoacylglycerophosphoserines & LPS \\
\hline & Glycerophosphoglycerophosphoglycerols & MLCL \\
\hline & Monomethylphosphatidylethanolamine & MMPE \\
\hline & Glycerophosphoethanolamine & NAPE \\
\hline & Diacylglycerophosphates & PA \\
\hline & Oxidized glycerophosphocholines & $\mathrm{PC}$ \\
\hline & Oxidized glycerophosphoethanolamines & PE \\
\hline & Glycerophosphoethanolamines & PE-NMe \\
\hline & Glycerophosphoethanolamines & PE-NMe2 \\
\hline & Glycerophosphoethanolamines & PEt \\
\hline & Diacylglycerophosphoglycerols & $P G$ \\
\hline & Diacylglycerophosphoglycerophosphates & PGP \\
\hline & Diacylglycerophosphoinositols & $\mathrm{Pl}$ \\
\hline & Diacylglycerophosphoinositolglycans & PIM1 \\
\hline & Glycerophosphoinositolglycans & PIM2 \\
\hline & Glycerophosphoinositolglycans & PIM3 \\
\hline & Glycerophosphoinositolglycans & PIM4 \\
\hline & Glycerophosphoinositolglycans & PIM5 \\
\hline & Glycerophosphoinositolglycans & PIM6 \\
\hline & Phosphatidylinositol mannoside inositol phosphate & PIMIP \\
\hline & Diacylglycerophosphoinositol monophosphates & PIP \\
\hline & Diacylglycerophosphoinositol bisphosphates & PIP2 \\
\hline & Glycerophosphoinositolbisphosphates & PIP2[3',4'] \\
\hline & Glycerophosphoinositolbisphosphates & PIP2[3',5'] \\
\hline & Glycerophosphoinositolbisphosphates & PIP2[4',5'] \\
\hline & Diacylglycerophosphoinositol trisphosphates & PIP3 \\
\hline & Glycerophosphoinositoltrisphosphates & PIP3[3', 4',5'] \\
\hline & Glycerophosphoinositolmonophosphates & $\mathrm{PIP}\left[3^{\prime}\right]$ \\
\hline & Glycerophosphoinositolmonophosphates & PIP[4] \\
\hline
\end{tabular}




\begin{tabular}{|c|c|c|}
\hline Category & Description & Abbreviation \\
\hline & $\begin{array}{l}\text { Glycerophosphoinositolmonophosphates } \\
\text { Diacylglyceropyrophosphates } \\
\text { Diacylglycerophosphoserines } \\
\text { Diacylglycerophosphoserines } \\
\text { Other Glycerophospholipids } \\
\text { Glycerophosphonocholines } \\
\text { Glycerophosphoinositolglycans } \\
\text { Diacylglycerophosphomonoradylglycerols }\end{array}$ & $\begin{array}{l}\text { PIP[5'] } \\
\text { PPA } \\
\text { PS } \\
\text { PS-NAc } \\
\text { PT } \\
\text { PnC } \\
\text { PnE } \\
\text { SLBPA }\end{array}$ \\
\hline $\begin{array}{l}\text { Saccharo- } \\
\text { lipids }\end{array}$ & $\begin{array}{l}\text { Acyltrehaloses } \\
\text { Acyltrehaloses } \\
\text { Acyltrehaloses } \\
\text { Acyltrehaloses }\end{array}$ & $\begin{array}{l}\text { AC2SGL } \\
\text { DAT } \\
\text { PAT16 } \\
\text { PAT18 }\end{array}$ \\
\hline $\begin{array}{l}\text { Sphingo- } \\
\text { lipids }\end{array}$ & $\begin{array}{l}\text { Glycosphingolipids } \\
\text { Glycosphingolipids } \\
\text { Acylceramides } \\
\text { Acylceramides } \\
\text { Acylceramides } \\
\text { Acylceramides } \\
\text { Acylceramides } \\
\text { Acylceramides } \\
\text { Acylceramides } \\
\text { Acylceramides } \\
\text { Acylceramides } \\
\text { Globoside } \\
\text { Globoside } \\
\text { Globoside } \\
\text { Glycosphingolipids } \\
\text { Ceramide-1-phosphates } \\
\text { N-acylsphingosines (ceramides) } \\
\text { Ceramide 1-phosphates } \\
\text { Glycosphingolipids } \\
\text { Ceramide phosphoethanolamines } \\
\text { Simple Glc series } \\
\text { Neutral glycosphingolipids } \\
\text { Glycosphingolipids } \\
\text { Acidic glycosphingolipids } \\
\text { Glycosphingolipids } \\
\text { Glycosphingolipids } \\
\text { Globoside } \\
\text { Globoside } \\
\text { Globoside } \\
\text { Globoside } \\
\text { Glycosphingolipids } \\
\text { Glycosphingolipids }\end{array}$ & $\begin{array}{l}\text { (3'-sulfo)LacCer } \\
\text { (Fuc)iGb3Cer } \\
\text { 1-O-behenoyl-Cer } \\
\text { 1-O-carboceroyl-Cer } \\
\text { 1-O-cerotoyl-Cer } \\
\text { 1-O-eicosanoyl-Cer } \\
\text { 1-O-lignoceroyl-Cer } \\
\text { 1-O-myristoyl-Cer } \\
\text { 1-O-palmitoyl-Cer } \\
\text { 1-O-stearoyl-Cer } \\
\text { 1-O-tricosanoyl-Cer } \\
\text { Ac-O-9-GD1a } \\
\text { Ac-O-9-GT1b } \\
\text { Ac-O-9-GT3 } \\
\text { Branched-Forssman } \\
\text { C1P } \\
\text { Cer } \\
\text { CerP } \\
\text { DSGG } \\
\text { EPC } \\
\text { FMC-5 } \\
\text { FMC-6 } \\
\text { Forssman } \\
\text { Fuc(Gal)-GM1 } \\
\text { Fuc(Gal)Gal- } \\
\text { iGb4Cer } \\
\text { Fuc-Branched- } \\
\text { Forssman } \\
\text { Fuc-GA1 } \\
\text { Fuc-GD1b } \\
\text { Fuc-GM1 } \\
\text { Fuc-GM1(NeuGc) } \\
\text { Fuc-iGb3Cer } \\
\text { FucGalGb3Cer }\end{array}$ \\
\hline
\end{tabular}




\begin{tabular}{|c|c|c|}
\hline Category & Description & Abbreviation \\
\hline & Glycosphingolipids & GA1 \\
\hline & Glycosphingolipids & GA2 \\
\hline & Neutral glycosphingolipids & GB4 \\
\hline & Glycosphingolipids & GD1 \\
\hline & Ganglioside GD1a(d18:1(4E)) & GD1a \\
\hline & Ganglioside GD1a alpha(d18:1(4E)) & GD1a alpha \\
\hline & Globoside & GD1a(NeuAc/NeuGc) \\
\hline & Globoside & GD1a(NeuGc/NeuAc) \\
\hline & Globoside & GD1a(NeuGc/NeuGc) \\
\hline & Ganglioside GD1b(d18:1(4E)) & GD1b \\
\hline & Ganglioside GD1c(d18:1(4E)) & GD1c \\
\hline & Globoside & GD1c(NeuGc/NeuGc) \\
\hline & Glycosphingolipids & GD2 \\
\hline & Glycosphingolipids & GD3 \\
\hline & Glycosphingolipids & GM1 \\
\hline & Globoside & GM1 alpha \\
\hline & Globoside & GM1(NeuGc) \\
\hline & Ganglioside GM1b(d18:1(4E)) & GM1b \\
\hline & Globoside & GM1b(NeuGc) \\
\hline & Glycosphingolipids & GM2 \\
\hline & Globoside & GM2(NeuGc) \\
\hline & Glycosphingolipids & GM3 \\
\hline & Gangliosides & GM4 \\
\hline & Glycosphingolipids & GP1 \\
\hline & Ganglioside GP1c(d18:1(4E)) & GP1c \\
\hline & Ganglioside GP1c alpha(d18:1(4E)) & GP1c alpha \\
\hline & Glycosphingolipids & GQ1 \\
\hline & Ganglioside GQ1b(d18:1(4E)) & GQ1b \\
\hline & Ganglioside GQ1b alpha(d18:1(4E)) & GQ1b alpha \\
\hline & Ganglioside GQ1c(d18:1(4E)) & GQ1c \\
\hline & Glycosphingolipids & GT1 \\
\hline & Ganglioside GT1a(d18:1(4E)) & GT1a \\
\hline & Ganglioside GT1a alpha(d18:1(4E)) & GT1a alpha \\
\hline & Ganglioside GT1b(d18:1(4E)) & GT1b \\
\hline & Globoside & GT1b alpha \\
\hline & Globoside & GT1b alpha(NeuGc) \\
\hline & Ganglioside GT1c(d18:1(4E)) & GT1c \\
\hline & Glycosphingolipids & GT2 \\
\hline & Glycosphingolipids & GT3 \\
\hline & Globoside & Gal(Fuc)-GA1 \\
\hline & Globoside & Gal(Fuc)-GD1b \\
\hline & Globoside & Gal-GD1b \\
\hline & Glycosphingolipids & Gal-iGb4Cer \\
\hline & Globoside & GalGal-GD1b \\
\hline & Glycosphingolipids & GalGalGalGb3Cer \\
\hline & Glycosphingolipids & GalGalGb3Cer \\
\hline
\end{tabular}




\begin{tabular}{|c|c|c|}
\hline Category & Description & Abbreviation \\
\hline & Globoside & $\begin{array}{l}\text { GalGalNAc- } \\
\text { GM1b(NeuGc) }\end{array}$ \\
\hline & Glycosphingolipids & GalGb3Cer \\
\hline & Glycosphingolipids & GalGb4Cer \\
\hline & Glycosphingolipids & $\begin{array}{l}\text { GalGlcNAc- } \\
\text { GalGb4Cer }\end{array}$ \\
\hline & Globoside & GalNAc-GD1a \\
\hline & Globoside & $\begin{array}{l}\text { GalNAc- } \\
\text { GD1a(NeuAc/NeuGc) }\end{array}$ \\
\hline & Globoside & $\begin{array}{l}\text { GalNAc- } \\
\text { GD1a(NeuGc/NeuAc) }\end{array}$ \\
\hline & Globoside & GalNAc-GM1 \\
\hline & Globoside & GalNAc-GM1b \\
\hline & Globoside & $\begin{array}{l}\text { GalNAc- } \\
\text { GM1b(NeuGc) }\end{array}$ \\
\hline & Globoside & $\begin{array}{l}\text { GalNAcGal(Fuc)- } \\
\text { GA1 }\end{array}$ \\
\hline & Glycosphingolipids & GalNAcGalGb3Cer \\
\hline & Glycosphingolipids & Gb3 \\
\hline & Glycosphingolipids & Gb3Cer \\
\hline & Glycosphingolipids & Gb4Cer \\
\hline & Glycosphingolipids & GlcNAc-GalGb4Cer \\
\hline & Glycosphingolipids & GlcNAcGb3Cer \\
\hline & Glycosphingolipids & Globo-A \\
\hline & Glycosphingolipids & Globo-B \\
\hline & Glycosphingolipids & Globo-H \\
\hline & Glycosphingolipids & Globo-Lex-9 \\
\hline & Glycosphingolipids & Hex2Cer \\
\hline & Neutral glycosphingolipids & Hex3Cer \\
\hline & Glycosphingolipids & HexCer \\
\hline & Ceramide phosphoinositols & IPC \\
\hline & Sphinganines & LCB \\
\hline & Sphingoid base 1-phosphates & LCBP \\
\hline & Hexosylsphingosine & LHexCer \\
\hline & Ceramides & LSM \\
\hline & Globoside & Lex-GM1 \\
\hline & Phosphosphingolipids & $\mathrm{M}(\mathrm{IP}) 2 \mathrm{C}$ \\
\hline & Phosphosphingolipids & MIPC \\
\hline & Glycosphingolipids & MSGG \\
\hline & Glycosphingolipids & NOR1 \\
\hline & Glycosphingolipids & NOR2 \\
\hline & Glycosphingolipids & NORint \\
\hline & Glycosphingolipids & $\begin{array}{l}\text { NeuAc(alpha2-6)- } \\
\text { MSGG }\end{array}$ \\
\hline & Glycosphingolipids & $\begin{array}{l}\text { NeuAc(alpha2-8)- } \\
\text { MSGG }\end{array}$ \\
\hline
\end{tabular}




\begin{tabular}{|c|c|c|}
\hline Category & Description & Abbreviation \\
\hline $\begin{array}{l}\text { Sphingo- } \\
\text { lipids }\end{array}$ & $\begin{array}{l}\text { Glycosphingolipids } \\
\text { Glycosphingolipids } \\
\text { Globoside } \\
\text { Glycosphingolipids } \\
\text { Glycosphingolipids } \\
\text { Globoside } \\
\text { Glycosphingolipids } \\
\text { Sulfoglycosphingolipids (sulfatides) } \\
\text { Ceramide phosphocholines (sphingomyelins) } \\
\text { Globoside } \\
\text { Globoside } \\
\text { Globoside } \\
\text { Glycosphingolipids } \\
\text { Glycosphingolipids } \\
\text { Glycosphingolipids } \\
\text { Glycosphingolipids } \\
\text { Glycosphingolipids } \\
\text { Glycosphingolipids } \\
\text { Glycosphingolipids } \\
\text { Glycosphingolipids }\end{array}$ & $\begin{array}{l}\text { NeuAcGal-iGb4Cer } \\
\text { NeuGc-GalGb4Cer } \\
\text { NeuGc-LacNAc- } \\
\text { GM1(NeuGc) } \\
\text { NeuGcNeuGc- } \\
\text { GalGb4Cer } \\
\text { Para-Forssman } \\
\text { SB1a } \\
\text { SHex2Cer } \\
\text { SHexCer } \\
\text { SM } \\
\text { SM1a } \\
\text { SM1b } \\
\text { SO3-GM1(NeuGc) } \\
\text { SO3-Gal-iGb4Cer } \\
\text { SO3-GalGb4Cer } \\
\text { SO3-Gb4Cer } \\
\text { SO3-iGb4Cer } \\
\text { SulfoGalCer } \\
\text { i-Forssman } \\
\text { iGb3Cer } \\
\text { iGb4Cer }\end{array}$ \\
\hline Sterols & $\begin{array}{l}\text { Sterol esters } \\
\text { Steryl esters } \\
\text { Desmosterol Ester } \\
\text { Ergostadienol Ester } \\
\text { Ergosterol Ester } \\
\text { Stigmasterol Ester } \\
\text { Lanosterol Ester } \\
\text { Sterols } \\
\text { Cholesterol and derivatives } \\
\text { Desmosterol } \\
\text { Ergostadienol } \\
\text { Ergosterol } \\
\text { Stigmasterol } \\
\text { Lanosterol }\end{array}$ & $\begin{array}{l}\text { SE } \\
\text { SE 27:1 } \\
\text { SE 27:2 } \\
\text { SE 28:2 } \\
\text { SE 28:3 } \\
\text { SE 29:2 } \\
\text { SE 30:2 } \\
\text { ST } \\
\text { ST 27:1;1 } \\
\text { ST 27:2;1 } \\
\text { ST 28:2;1 } \\
\text { ST 28:3;1 } \\
\text { ST 29:2;1 } \\
\text { ST } 30: 2 ; 1\end{array}$ \\
\hline Polyketides & $\begin{array}{l}\text { Anacardic acids and derivatives } \\
\text { Alkyl catechols and derivatives } \\
\text { Alkyl phenols and derivatives } \\
\text { Alkyl resorcinols and derivatives }\end{array}$ & $\begin{array}{l}\text { ANACARD } \\
\text { CATECHOL } \\
\text { PHENOL } \\
\text { RESORCINOL }\end{array}$ \\
\hline
\end{tabular}




\section{Acronyms}

API application programming interface. 6,7

CLI command line interface. 20

Goslin grammar of succinct lipid nomenclatures. 2, 3, 6, 9, 12, 16, 19, 23

HTTP hypertext transfer protocol. 6, 7

JDK Java Development Kit. 19

JRE Java Runtime Environment. 19

JSON JavaScript object notation. 6

LCB long chain base. 7

REST representational state transfer. 6, 7

\section{References}

[1] Fahy, E.; Subramaniam, S.; Murphy, R. C.; Nishijima, M.; Raetz, C. R. H.; Shimizu, T.; Spener, F.; van Meer, G.; Wakelam, M. J. O.; Dennis, E. A. Update of the LIPID MAPS comprehensive classification system for lipids. Journal of Lipid Research 2009, 50, S9-S14.

[2] Aimo, L.; Liechti, R.; Hyka-Nouspikel, N.; Niknejad, A.; Gleizes, A.; Götz, L.; Kuznetsov, D.; David, F. P.; van der Goot, F. G.; Riezman, H.; Bougueleret, L.; Xenarios, I.; Bridge, A. The SwissLipids knowledgebase for lipid biology. Bioinformatics 2015, 31, 2860-2866.

[3] Wishart, D. S. et al. HMDB: the Human Metabolome Database. Nucleic Acids Research 2007, 35, D521-D526.

[4] Liebisch, G.; Vizcaíno, J. A.; Köfeler, H.; Trötzmüller, M.; Griffiths, W. J.; Schmitz, G.; Spener, F.; Wakelam, M. J. O. Shorthand notation for lipid structures derived from mass spectrometry. Journal of Lipid Research 2013, 54, 1523-1530. 